\title{
Chaotic and Periodic Natural Convection for Moderate and High Prandtl Numbers in a Porous Layer subject to Vibrations
}

\author{
by \\ Johnathan J. Vadasz ${ }^{\text {a, }}$, Josua P. Meyer ${ }^{\text {a }}$, Saneshan Govender ${ }^{\text {b }}$ \\ ${ }^{a}$ Department of Mechanical and Aeronautical Engineering, University of Pretoria, Lynnwood Rd., Hatfield, \\ Pretoria 0002, South Africa \\ ${ }^{\mathrm{b}}$ Gas Division EED, ESKOM, Arivia Business Park, Building 1, Simba Road, Sunninghill, Johannesburg \\ 2000, South Africa
}

( Submitted: November 30, 2013)

\begin{abstract}
The analysis of natural convection for moderate and high Prandtl numbers in a fluid saturated porous layer heated from below and subject to vibrations is presented with a twofold objective. First, it aims at investigating the significance of including a time derivative term in Darcy's equation when wave phenomena are being considered. Second it is dedicated to reporting results related to the route to chaos for moderate and high Prandtl number convection. The results present conclusive evidence indicating that the time derivative term in Darcy's equation cannot be neglected when wave phenomena are being considered even when the coefficient to this term is extremely small. The results also show occasional chaotic "bursts" at specific values (or small range of values) of $R$, exceeding some threshold. This behavior is quite distinct from the case without forced vibrations, when the chaotic solution occupies a wide range of $R$ values, interrupted only by periodic "bursts". Periodic and chaotic solution alternate as the value of the scaled Rayleigh number varies.
\end{abstract}

Keywords: Natural Convection, Vibrations, Prandtl Number, Chaos.

\footnotetext{
${ }^{*}$ Corresponding author.

E-mail addresses: Johnathan@DrVadasz.com (J.J. Vadasz), Josua.Meyer@up.ac.za (J.P. Meyer), Saneshan.Govender@eskom.co.za (S. Govender).
} 


\section{Nomenclature}

\section{Latin Symbols}

$D a=$ Darcy number, defined by $k_{*} / L_{*}^{2}$.

$\hat{\boldsymbol{e}}_{x}=$ unit vector in the $x$ direction.

$\hat{\boldsymbol{e}}_{z}=$ unit vector in the $\mathrm{z}$ direction.

$\hat{\boldsymbol{e}}_{n}=$ unit vector normal to the boundary, positive outwards.

$g_{*}=$ acceleration due to gravity.

$H_{*}=$ the height of the layer.

$k_{*}=$ permeability of the porous domain.

$L_{*}=$ the length of the porous domain.

$L=$ reciprocal of aspect ratio, equals $L_{*} / H_{*}$.

$p=$ reduced pressure (dimensionless).

$\operatorname{Pr}=$ Prandtl number, equals $v_{*} / \alpha_{e^{*}}$.

$R a=$ porous media Rayleigh number, equals $\beta_{*} \Delta T_{c} g_{*} k_{*} H_{*} / \alpha_{e^{*}} \nu_{*}$.

$R=$ scaled Rayleigh number, equals $R a / 4 \pi^{2}$.

$t=$ time (dimensionless)

$T=$ dimensionless temperature, $\left(T-T_{C}\right) /\left(T_{H}-T_{C}\right)$.

$T_{C}=$ cold wall temperature

$T_{H}=$ hot wall temperature.

$u=$ horizontal $x$ component of the filtration velocity.

$w=$ vertical $z$ component of the filtration velocity.

$x=$ horizontal length co-ordinate.

$z=$ vertical co-ordinate.

$X=$ rescaled amplitude.

$Y=$ rescaled amplitude.

$Z$ = rescaled amplitude.

\section{Greek Symbols}

$\alpha=$ a parameter related to the time derivative term in Darcy's equation.

$\alpha_{e^{*}}=$ effective thermal diffusivity.

$\beta_{*}=$ thermal expansion coefficient. 
$\gamma=$ a parameter defined by $L^{2} /\left(L^{2}+1\right)$

$\phi=$ porosity

$\nu_{*}=$ fluid's kinematic viscosity.

$\psi=$ stream function.

$\Delta T_{c}=$ characteristic temperature difference, equals $\left(T_{H}-T_{C}\right)$.

$\omega=$ forced vibration frequency.

$\delta=$ forced vibration amplitude

\section{Subscripts}

$*=$ dimensional values.

$c r=$ critical values.

$C=$ related to the cold wall.

$H=$ related to the hot wall.

\section{Introduction}

The effect of vibrating a fluid saturated porous layer heated from below on the consequent natural convection developing inside the layer is analyzed for moderate and high Prandtl numbers. Traditional applications of convection in porous media are associated with moderate values of Prandtl number with the resulting effect of a very small coefficient to the time derivative in Darcy's equation allowing usually the neglect of the time derivative term. Nevertheless, when wave effects are of interest it was suggested to include the time derivative in Darcy's equation as demonstrated by Vadasz (1998) and Vadasz and Olek (2000). In modern applications of heat and mass transfer in porous media the Prandtl number can vary over a wide order of magnitude range. Comprehensive reviews of the fundamentals of heat convection in porous media are presented by Nield and Bejan (2006) and Bejan (1995).

The objective of the present paper is twofold. First, it investigates the significance of including a time derivative term in Darcy's equation when wave phenomena are being considered. In the present case there is an apparent good reason for the neglect of the time derivative term, since its coefficient is very small $\left(\sim 10^{-3}\right.$ ). Nevertheless, the results evidently show that due to the forced vibrations the solution is periodic, quasiperiodic, or chaotic, and therefore it departs from the plane consistent with the assumption of ignoring the time derivative term. Second, the paper is dedicated to reporting results related to the route to chaos for moderate and high Prandtl number convection in a porous layer heated from below and subject to vibrations. The dimensionless group forming the coefficient to the time derivative term in the Darcy equation is $1 / \chi$, 
where $\chi=\phi \operatorname{Pr} / D a$ was named the Vadasz number by Straughan (2001, 2004), followed by Lombardo and Mulone (2002), Mulone and Straughan (2006), Sheu (2006), Bhadauria (2007), Mahmud and Hashim (2011), Roslan, Mahmud and Hashim (2011), Bhadauria and Kiran (2013) and more. It is only through this combined dimensionless group that Prandtl number is affecting the flow in porous media. Hence, while $\operatorname{Pr}$ can take values from as small as $10^{-3}$ for liquid metals and up to $10^{3}$ for oils, the corresponding values for $\chi$ will be magnified by a factor of $\phi / D a$ which is typically a big number. This factor can take values from 10 to $10^{20}$. Therefore the values of $\chi$ can be expected in the range from $10^{-2}$ to $10^{23}$. In traditional applications of transport phenomena in porous media typical values of $\chi$ are quite big, a fact that provides the justification for neglecting the time derivative term in Darcy's equation. However, when wave phenomena are of interest the time derivative term is expected to be included in order to prevent the reduction of the order of the system in the time domain. The effect of including this term in the equations on the results will be observed.

Govender (2004) presented a linear stability for natural convection in a vertically modulated porous layer subject to constant vertical stratification and obtained a Mathieu equation that was solved in terms of Mathieu functions. The analysis of the transition point from synchronous to sub-harmonic solutions was investigated by Govender (2005a). Govender (2005b) showed that a vibrating fluid-saturated porous layer can be destabilized even when heated from above as the gravity modulation due to vibrations may on average overcome the opposite stabilizing effect due to gravity (heating from above). As the results represent linear stability solutions, the amplitude of convection is not obtained. To find the unknown amplitude of convection Govender (2005c) presented results where the amplitude of convection may be evaluated using the assumption of a "frozen time", which might be applicable for large time scales and consequently small frequencies. In addition, weak non-linear solutions, which provide information about the convection amplitude near the convection threshold, were presented by Govender (2005d). A comprehensive review of all these results was presented by Govender (2008). The theoretical results reported above usually correspond to a rectangular two-dimensional layer. As distinct from the rectangular geometries considered previously, a cylindrical vibrating porous layer was considered by Govender (2006) in producing results of linear stability, while the stability of solutal convection in a cylindrical mushy layer subject to vibrations was presented by Govender (2011). A combination of rotation and vibrations on convection in a porous layer far away from the axis of rotation was presented by Govender (2010). Elhajjar et al. (2009) analyzed the effect of smallamplitude and high-frequency vertical vibrations on the separation of a binary mixture saturating a shallow horizontal porous layer heated from below. They applied direct numerical simulations and linear stability analysis to the two-dimensional equations averaged over the period of the impressed vibrations. The averaging method adopted by Elhajjar et al. (2009) can be justified for thermal vibrational convection in the limit of small-amplitude and high-frequency vibrations. The method is similar to the one adopted by Bardan and Mojtabi (2000), for investigating the convective instability in a porous layer subject to vertical vibration, 
or by Charrier-Mojtabi et al. (2007) dealing with Soret-driven convection in a horizontal porous layer. Jounet and Bardan (2001) adopted a similar averaging method to investigate the onset of thermohaline convection in a rectangular porous cavity subject to vibrations. They performed a linear stability analysis, a weak non-linear analysis as well as a direct numerical simulation to confirm and illustrate their findings. Jounet and Bardan (2001) focused on the analysis of the stationary bifurcation, which as expected turned out to be of a pitchfork type. They also concluded that depending on the governing parameters, vibrations could delay or promote the onset of convection. An extensive review of computational heat convection in porous media with applications among others to alloy solidification was conducted by Vafai and Hadim (2000). Vadasz and Olek (1999, 2000) and Vadasz (1999 a, b, 2001) developed a spectral method of analyzing heat convection problems beyond the neighborhood of the convection onset. Essentially, the method uses the eigenfunctions obtained from the weak non-linear analysis to yield a truncated system of ordinary differential equations representing the dynamics of the convection process, i.e. its behavior in time. This system was shown to be similar to the Lorenz system (Lorenz, 1963; Sparrow, 1982). More recently Mahmud and Hashim (2010, 2011), and Roslan et al. (2011) showed that this system could be controlled by using feedback control methods in order to promote or delay the transition from laminar to weak turbulent convection. Their results were extended by Magyari (2010a) and Vadasz (2010), while Magyari (2010b) showed how this system could be seen as a system of coupled oscillators.

Vadasz, Meyer and Govender (2013) have shown that forced vibrations render an otherwise chaotic solution into a periodic or quasi-periodic one. This might suggest the rendering of the subcritical bifurcation into a supercritical one. Their study focused on values of $\chi \sim 100$, which correspond to small and moderate Prandtl numbers. As distinct, the present paper focuses on moderate and high values of Prandtl number and follows the twofold objectives listed above, i.e. (a) it investigates the significance of including a time derivative term in Darcy's equation when wave phenomena are being investigated and, (b) it reports results related to the route to chaos for moderate and high Prandtl number convection in a porous layer heated from below and subject to vibrations.

\section{Problem Formulation}

Following the derivations presented by Vadasz, Meyer and Govender (2013) for a two-dimensional rectangular fluid saturated porous layer heated from below and subject to vibrations as presented in Figure 1, one may present the governing equations in the following dimensionless form

$$
\begin{aligned}
& {\left[\frac{1}{\chi} \frac{\partial}{\partial \tilde{t}}+1\right] \nabla^{2} \psi+R a[1+\delta \sin (\tilde{\omega} \tilde{t})] \frac{\partial T}{\partial x}=0} \\
& \frac{\partial T}{\partial \tilde{t}}+\frac{\partial \psi}{\partial z} \frac{\partial T}{\partial x}-\frac{\partial \psi}{\partial x} \frac{\partial T}{\partial z}=\nabla^{2} T
\end{aligned}
$$


where $R a=\beta_{*}\left(T_{H}-T_{C}\right) g_{*} k_{*} H_{*} / \alpha_{e^{*}} \nu_{*}$ is the porous media Rayleigh number, $\psi$ is the stream function defined by $u=\partial \psi / \partial x, w=-\partial \psi / \partial z, \tilde{t}$ is dimensionless time, $\delta$ and $\tilde{\omega}$ are the amplitude and frequency of the forced vibrations, and $\chi=\phi P r / D a$. While $\operatorname{Pr}$ can take values from as small as $10^{-3}$ for liquid metals and up to $10^{3}$ for oils, the corresponding values for $\chi$ will be magnified by a factor of $\phi / D a$ which is typically a big number. This factor can take values from 10 to $10^{20}$. Therefore the values of $\chi$ can be expected in the range from $10^{-2}$ to $10^{23}$.

Vadasz, Meyer and Govender (2013) focused their study on a value of $\chi \sim 100$, which corresponds to small and moderate Prandtl numbers, while the present study deals with a value of $\chi \sim 10,000$, which corresponds to moderate and large values of Prandtl number. For such large values of $\chi$ the neglect of the time derivative term is justified, except that in the present case the oscillatory nature of the solution indicates that such a neglect will cause a major flaw in the solution.

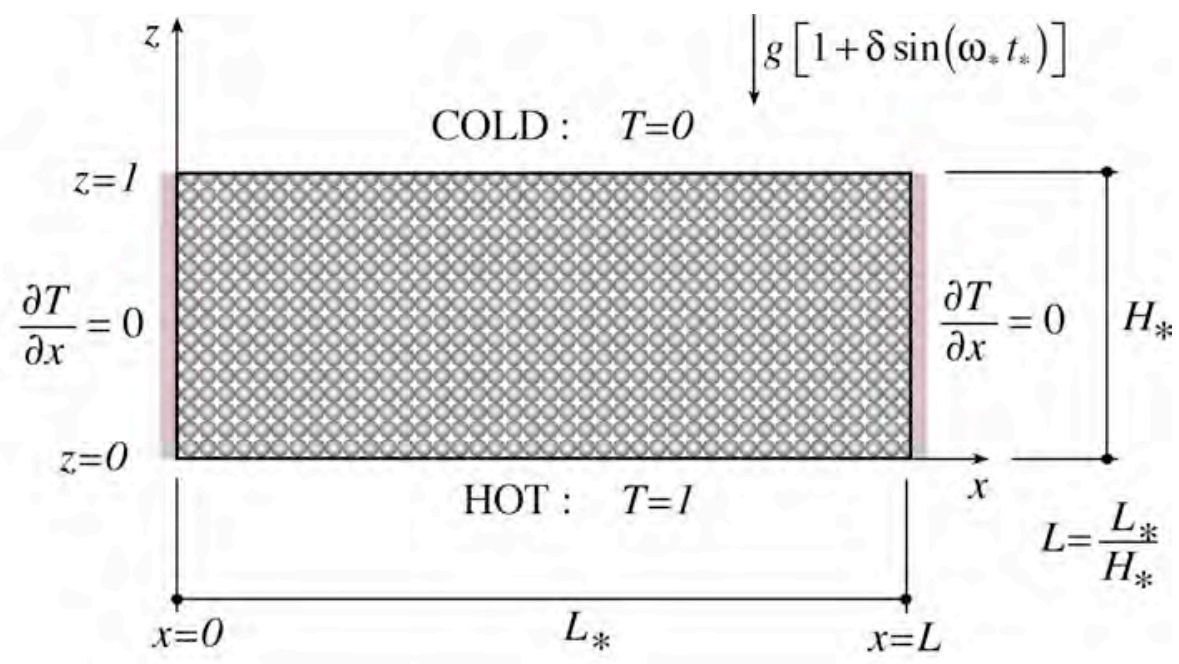

Figure 1: Problem formulation for a two-dimensional porous domain heated from below subject to accelerations due to gravity and vibrations.

\section{Method of Solution and Analysis}

A spectral method of solution is used by considering the truncated expansion based on Vadasz and Olek (1999, 2000), Magyari (2010, Vadasz, Meyer and Govender (2013)

$$
\begin{aligned}
& \psi=A_{11} \sin \left(\frac{\pi x}{L}\right) \sin (\pi z) \\
& T=1-z+B_{11} \cos \left(\frac{\pi x}{L}\right) \sin (\pi z)+B_{02} \sin (2 \pi z)
\end{aligned}
$$


that can be considered equivalent to a Galerkin expansion of the solution in both the $x$ and $z$ directions, truncated when $(i+j)=2$, where $i$ is the Galerkin summation index in the $x$ direction while $j$ is the Galerkin summation index in the $z$ direction. Substituting expansions (3) and (4) into the governing equations (1) and (2), multiplying the resulting equations by the corresponding eigenfunctions and integrating over the domain, produces a system of ordinary differential equations for the amplitudes $A_{11}(\tilde{t}), B_{11}(\tilde{t}), B_{02}(\tilde{t})$ . By introducing for convenience the following notation

$\gamma=\frac{L^{2}}{L^{2}+1} ; \quad \theta=\frac{L}{\gamma}=\frac{L^{2}+1}{L}$

and by rescaling the time variable and the forced frequency in the form

$t=\frac{\pi^{2}}{\gamma} \tilde{t}$

$\omega=\frac{\tilde{\omega} \gamma}{\pi^{2}}$

one may present the resulting equations after multiplying them by $\gamma / \pi^{2}$ and rescaling the amplitude with respect with their corresponding positive fixed points associated with the corresponding autonomous system, in the form

$X=-\frac{A_{11}}{2 \sqrt{2} \gamma^{1 / 2} \theta(R-1)^{1 / 2}}$

$Y=\frac{\pi R B_{11}}{2 \sqrt{2} \gamma^{1 / 2}(R-1)^{1 / 2}}$

$Z=-\frac{\pi R}{(R-1)} B_{02}$

leading to the following three ordinary differential equations for the amplitudes of convection

$$
\begin{aligned}
& \frac{1}{\alpha} \frac{\mathrm{d} X}{\mathrm{~d} t}=X-[1+\delta \sin (\omega t)] Y \\
& \frac{\mathrm{d} Y}{\mathrm{~d} t}=-Y+R X-(R-1) X Z \\
& \frac{\mathrm{d} Z}{\mathrm{~d} t}=4 \gamma(X Y-Z)
\end{aligned}
$$

where

$$
R=\frac{R a}{\pi^{2}} \text { and } \alpha=\frac{\chi \gamma}{\pi^{2}}
$$


are the scaled Rayleigh number, and the scaled coefficient to the time derivative term, respectively. For the solution of the non-autonomous system (12), (13), (14) one introduces an additional dummy dependent variable $W$ in the form

$W=\omega t$

leading to the following system

$$
\begin{aligned}
& \dot{X}=-\alpha(X-Y)+\alpha \delta Y \sin (W) \\
& \dot{Y}=-Y+R X-(R-1) X Z \\
& \dot{Z}=4 \gamma(X Y-Z) \\
& \dot{W}=\omega
\end{aligned}
$$

where the Newtonian notation for time derivatives was introduced, i.e. $\dot{X}=\mathrm{d} X / \mathrm{d} t, \dot{Y}=\mathrm{d} Y / \mathrm{d} t$, $\dot{Z}=\mathrm{d} Z / \mathrm{d} t$, and $\dot{W}=\mathrm{d} W / \mathrm{d} t$.

In order to observe the effect of the neglect of the time derivative term in the original Darcy equation when the value of $\chi$ is very large $(\chi>>1)$ one may consider equations (11), (12) and (13) for the case when $\alpha$ is very large $(\alpha>>1)$ as the value of $\gamma$ consistent with the present problem is $\gamma=0.5$. Then, with $\alpha>>1$ the neglect of the time derivative term in equation (11) can be justified, leading to the approximation

$$
X=[1+\delta \sin (\omega t)] Y
$$

When there are no forced vibrations $\omega=0$ and this equation becomes

$Y=X$

implying that the solution that is consistent with the neglect of the time derivative term in the case of no forced vibrations lies on the plane $Y=X$ in the three dimensional $X-Y-Z$ phase space. Its projection on the $X-Y$ plane will be the straight line $Y=X$.

\section{Results and Discussion}

Equations (16), (17), (18), and (19) were solved numerically to double precision by using the fifth and sixth order Runge-Kutta-Verner method from the IMSL Library (DIVPRK) (Hull, 1976) up to the desired accuracy by controlling a tolerance control parameter. The initial conditions used for all numerical solutions were

$t=0: X=Y=Z=0.9 ; W=0$

and the parameter values for all numerical solutions were

$\gamma=0.5 ; \alpha=500 ; \delta=1$

while the values of $R$ and $\omega$ were varied to observe the impact of the vibrations on the solution. 
It is anticipated that a strong forcing vibration amplitude $\delta$ will affect the convection, however, the more important point is to test whether specific frequencies may resonate the solution even when the forcing vibration amplitude $\delta$ is not strong. Therefore, $\delta$ was kept constant in all the numerical computations and was assigned a value of 1 as shown in equation (23). The value of $\gamma=0.5$ is consistent with the steady convection wavenumber at $R=1$, while $\alpha=500$ is consistent with $\chi \sim 10,000$, and consequently moderate and high Prandtl numbers.

The visualization of the solution for $W(t)$ does not add anything to the graphical description because by its own definition $W(t)=\omega t$. Therefore there is no point in including $W$ in the presentation, and only the amplitudes $X(t), Y(t)$ and $Z(t)$ are represented graphically. A large number of computational results were obtained. Only a select group of these results is presented here to demonstrate the types of different solutions obtained. For reference purposes, the first result presented in each group corresponds to the solution obtained for the case when there is no vibration. No vibration was obtained by setting the frequency $\omega=0$. The solution is presented graphically first in the form of the solution amplitude $X(t)$ plotted against time up to a long time value of $t=210$ and includes the transient too. Then, three additional graphs are presented for each combination of $R$ and $\omega$ values. These graphs represent on the phase diagram the projection of the solution on the $X-Y, X-Z$, and $Y-Z$ planes. The data used to present the solution on the phase diagram consisted only of the post-transient solution. All solutions with the exception of Figure 2 are plotted such that the data points are not connected, even when in some plots they do seem connected.

The numerical results for the case without forced vibrations $\omega=0$ serves as a reference and is presented in Figure 2, which includes the transient part of the solution. It represents the solution for a value of $R=75$. At this $R$ value the solution decays to the fixed point $X=Y=Z=1$ as observed from Figure 2a,b,c,d. A particularly important conclusion can be drawn from Figure $2 b$, representing the projection of the solution on the $X-Y$ plane. It is evident form this figure that the solution even during the transient lies on the straight line $Y=X$, representing the approximation associated with the neglect of the time derivative term in the original Darcy equation. However, as soon as the value of $\omega$ becomes nonzero this result is bound to change drastically, as observed from Figure 3 where $\omega=5$, while the value of $R$ is still the same, i.e. $R=75$. The figure shows a clearly periodic solution. The data points are not connected despite the apparent visual form. Figure $3 \mathrm{~b}$ shows a clear deviation of the solution from the straight line $Y=X$, representing the approximation associated with the neglect of the time derivative term in the original Darcy equation. Obviously, neglecting the time derivative term in the equation would have caused a flawed solution, and not an approximation as one would have anticipated. The solution for the same value of $R=75$ and for $\omega=7$ is presented in Figure 4, identifying a periodic solution. The deviation of the solution from the straight line 
$Y=X$ (the dashed line in Figure 4b) is even more profound then in the previous graph. For this combination of parameters ( $R=75$ and $\omega=7$ ) one may observe from the figure that solution spends a long time in the neighborhood $X=Y=0$ until it is repelled from this neighborhood. Another periodic solution is obtained for a larger frequency, i.e. $\omega=45$ and the same value of $R=75$, as presented in Figure 5 (the data points are not connected despite the apparent visual form). So far no chaotic solutions were obtained. In order to find out at what values of $\omega$ and $R$ we may anticipate chaotic solutions we evaluated the Lyapunov exponents as a function of $R$ for different values of $\omega$. Accordingly, we were able to identify that for $R=75$ a chaotic solution would be obtained at $\omega=6.2$, as for this parameter combination at least one Lyaounov exponent is poisitive. The results for $R=75$ and $\omega=6.2$ are presented in Figure 6 showing a typical chaotic cloud. Similarly Figure 7 shows graphically a chaotic solution for $R=110$ and $\omega=10$. The results for $R=143$ and $\omega=10$ are presented in Figure 8, where the fact that solution is chaotic is not so obvious. It was established from the fact that one of the Lyapunov exponents was positive. Figure $8 \mathrm{~b}$ also shows the deviation of the solution from the straight line $Y=X$ (the dashed line in Figure 8b) representing the approximation associated with the neglect of the time derivative term. Figures 9 and 10 are the graphical representation of the Lyapunov exponents as a function of $R$ for values of $\omega=13$ and $\omega=45$, respectively. From these graphs one can observe the occasional chaotic "bursts" at specific values (or small range of values) of $R$, exceeding some threshold. This behavior is quite distinct from the case without forced vibrations, when the chaotic solution occupied a wide range of $R$ values, interrupted only by periodic "bursts".

\section{Conclusions}

The effect of vibrating a fluid saturated porous layer heated from below on the consequent natural convection developing inside the layer was analyzed for moderate and high Prandtl numbers. It can be definitely concluded that the neglect of the time derivative in the original Darcy equation is not justified when vibrations and wave effects are being considered, even when the coefficient to this term is extremely small as

in the present paper $\left(10^{-4}\right)$. The results provide definite evidence for the departure of the solution from the one expected when such an approximation is applied. The results also show that periodic solutions and chaotic solutions alternate as the value of the scaled Rayleigh number changes, when forced vibrations are present.

\section{Acknowledgments}

The University of Pretoria and Prof. JP. Meyer gave much support for this project, both financially and intellectually. One of the authors (JJV) wishes to thank them for all the support. 
Figure 2
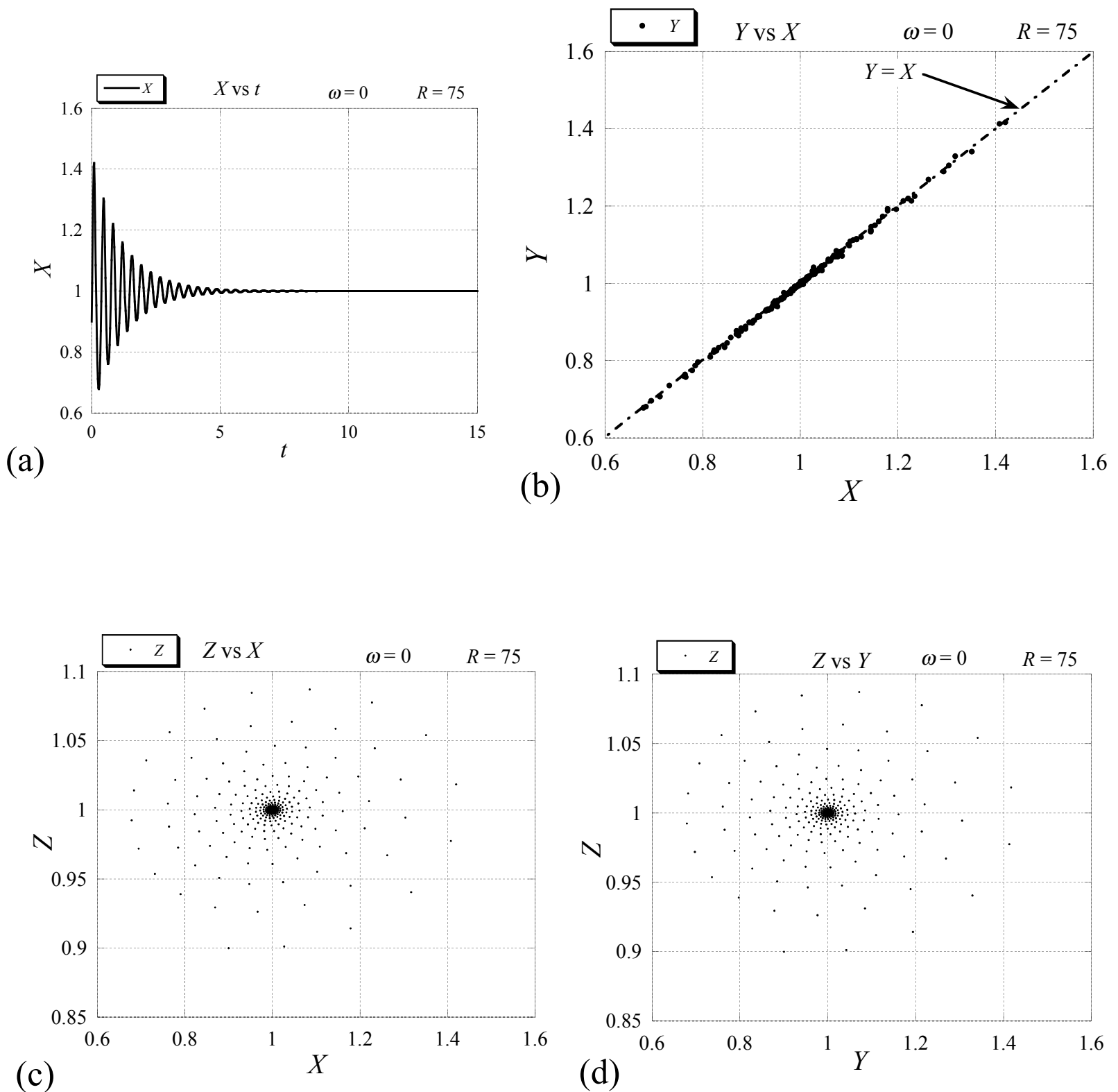

Figure 2: Graphical description of the solution for $\omega=0$ and $R=75$; (a) the amplitude solution in the time domain $X(t)$; (b), (c), and (d) the solution on the phase diagram: projection of the trajectory on the $X-Y, X-Z$, and $Y-Z$ planes, respectively. 


\section{Figure 3}

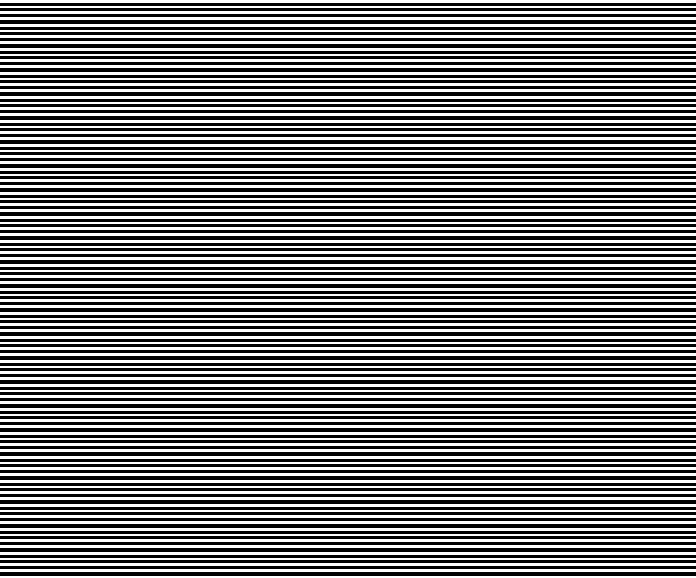

(a)

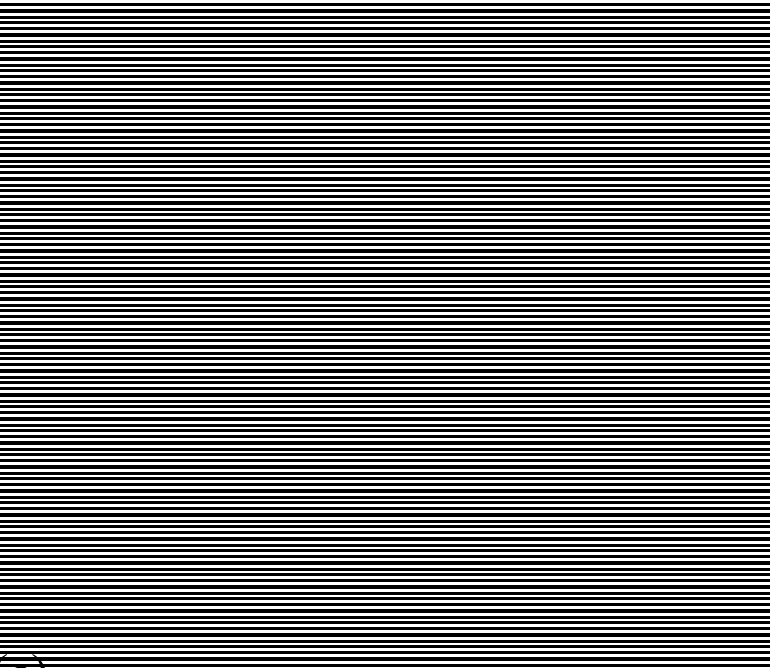

(c) (d)

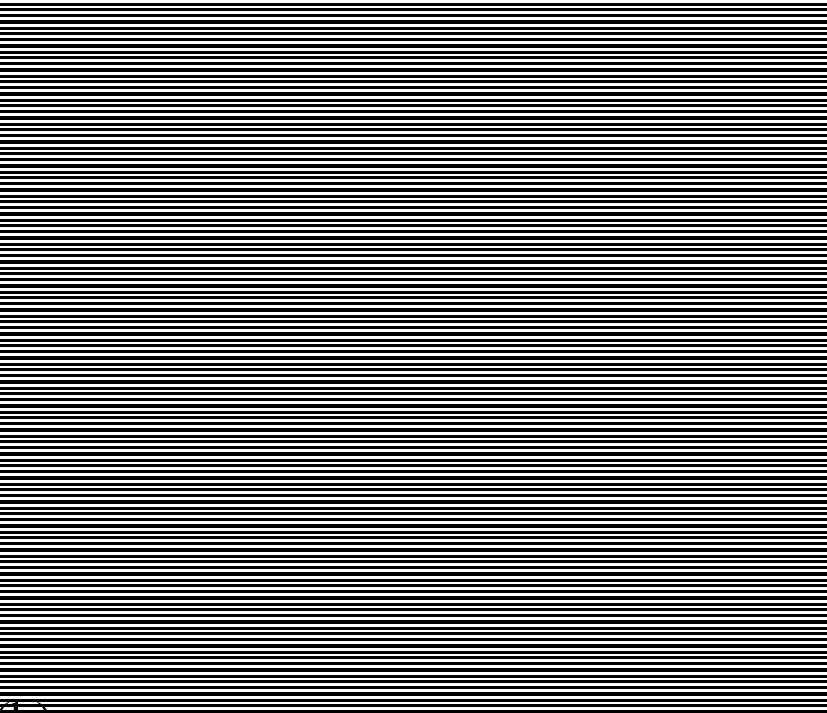

(b)

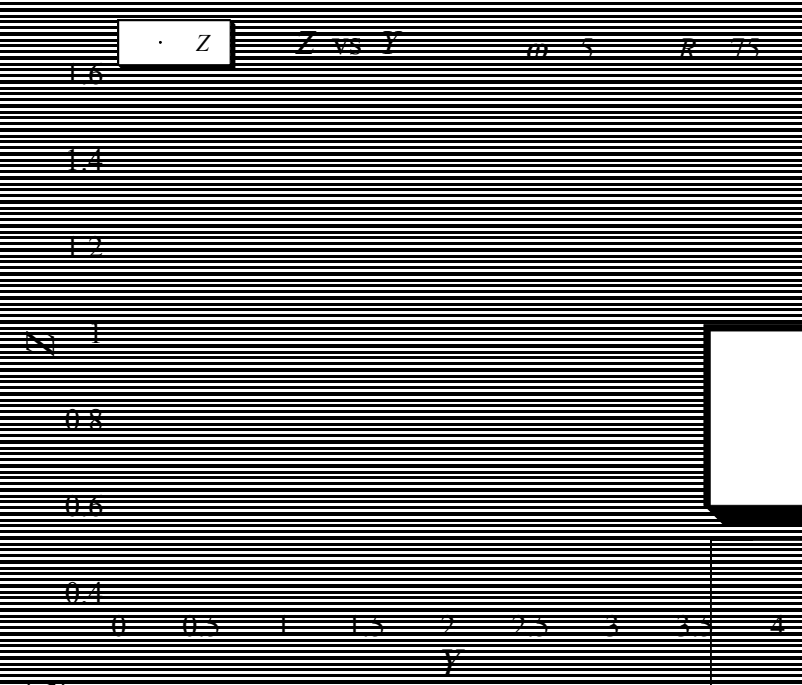

Figure 3: Graphical description of the solution for $\omega=5$ and $R=75$; (a) the amplitude solution in the time domain $X(t)$; (b), (c), and (d) the solution on the phase diagram: projection of the trajectory on the $X-Y, X-Z$, and $Y-Z$ planes, respectively. 


\section{Figure 4}

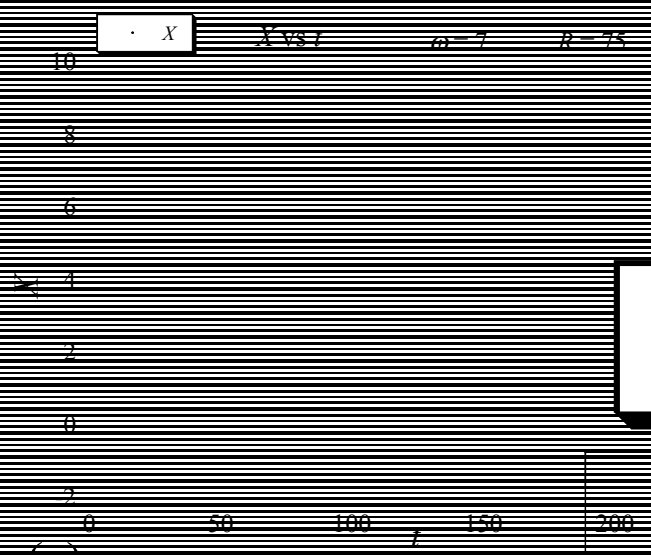

(a)

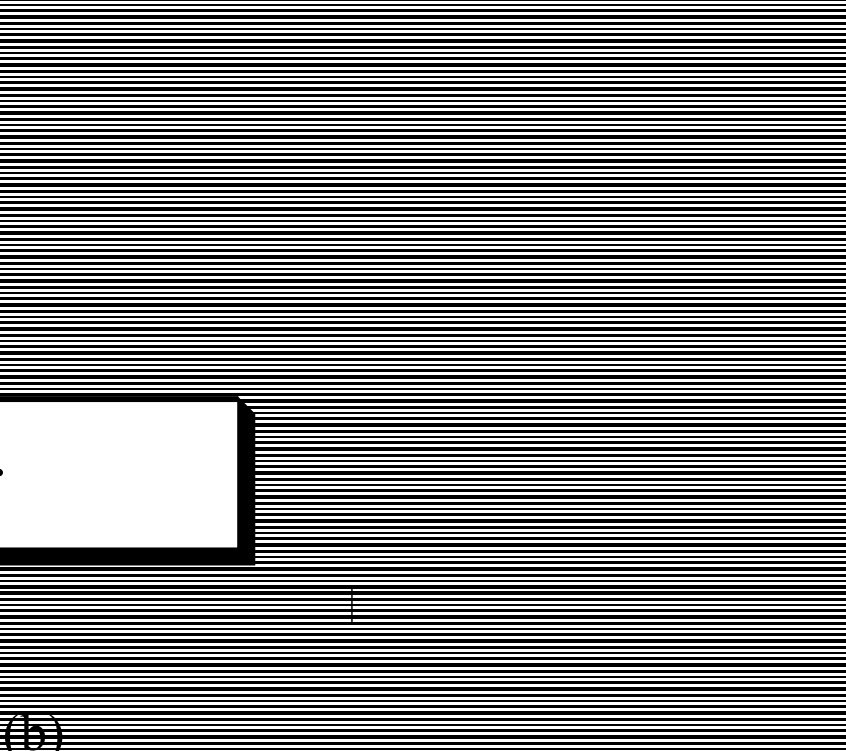

(b)

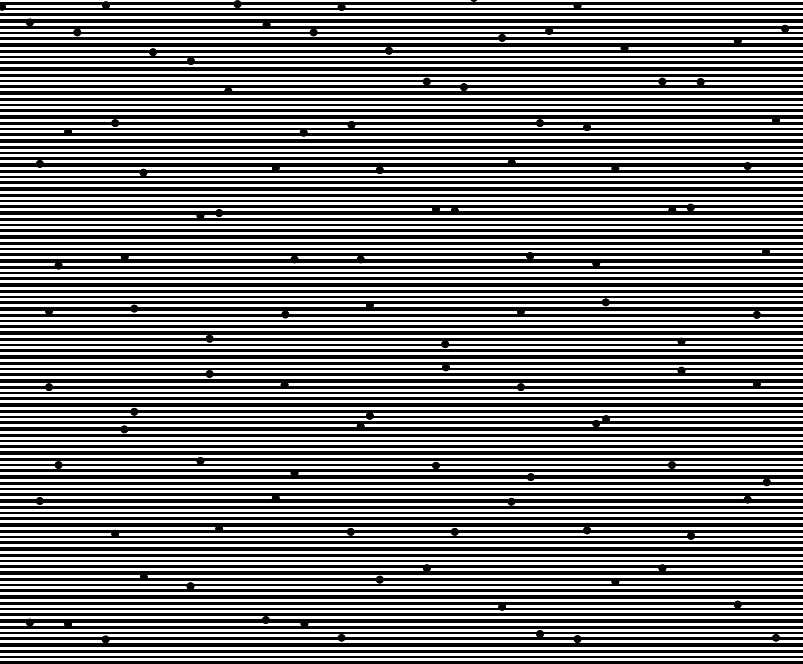

(d)

Figure 4: Graphical description of the solution for $\omega=7$ and $R=75$; (a) the amplitude solution in the time domain $X(t)$; (b), (c), and (d) the solution on the phase diagram: projection of the trajectory on the $X-Y, X-Z$, and $Y-Z$ planes, respectively. 


\section{Figure 5}

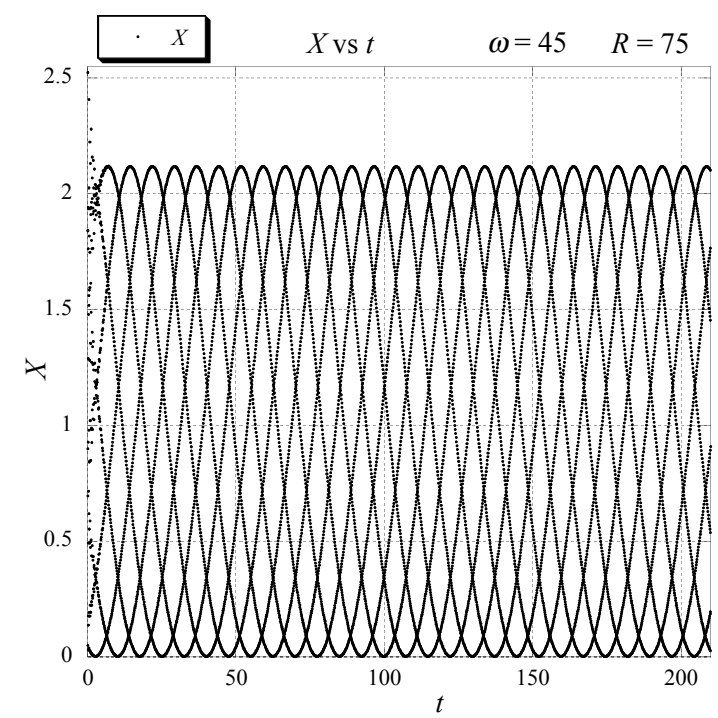

(a)

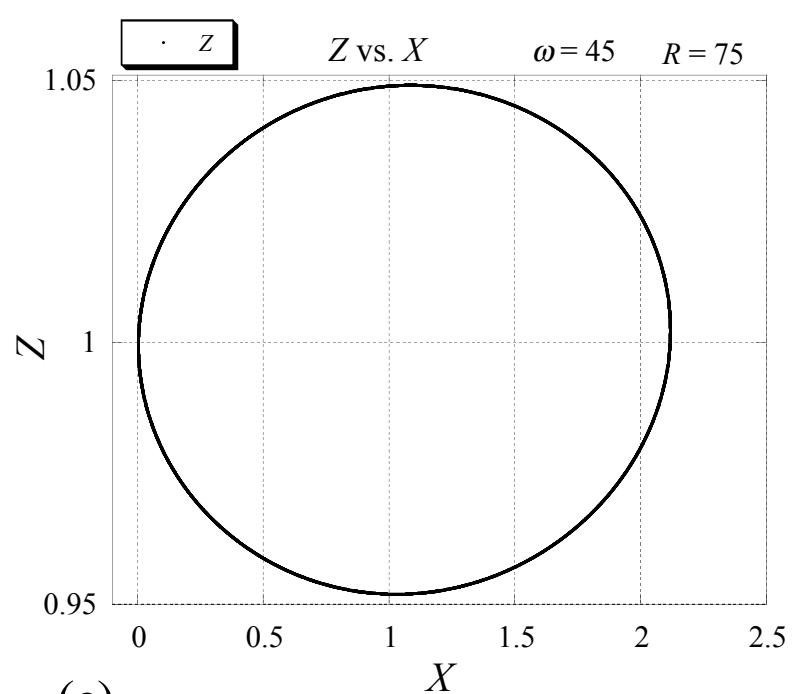

(c)

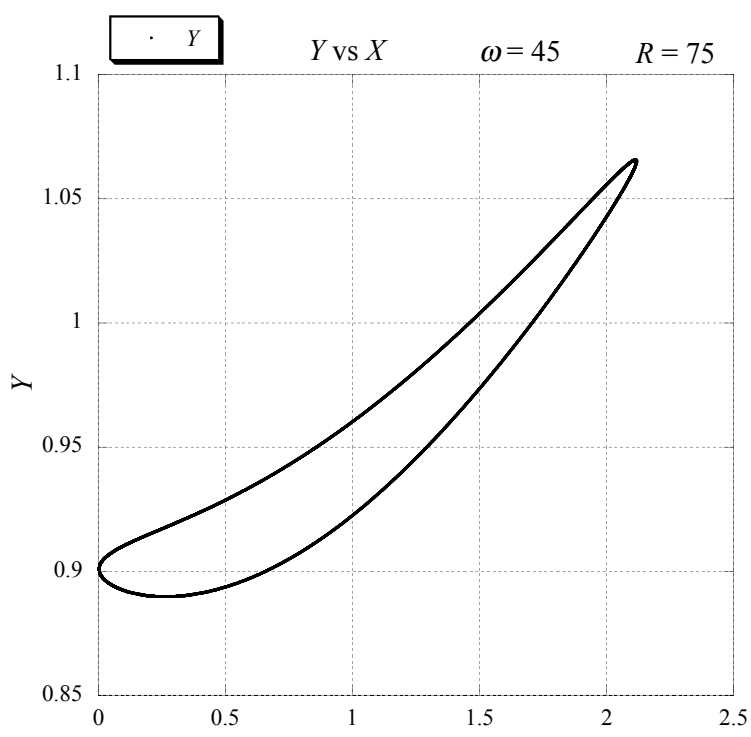

(b)

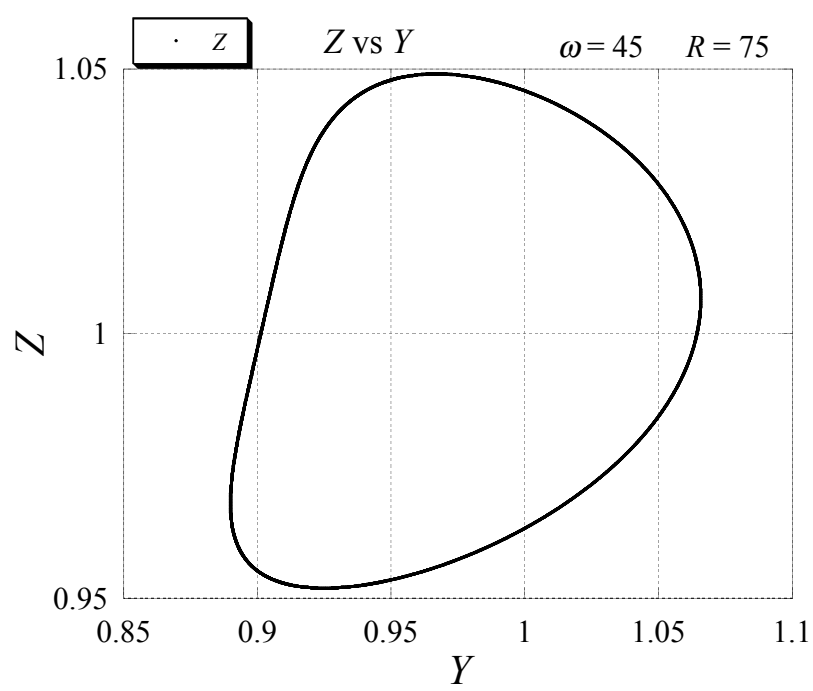

(d)

Figure 5: Graphical description of the solution for $\omega=45$ and $R=75$; (a) the amplitude solution in the time domain $X(t)$; (b), (c), and (d) the solution on the phase diagram: projection of the trajectory on the $X-Y, X-Z$, and $Y-Z$ planes, respectively. 
Figure 6

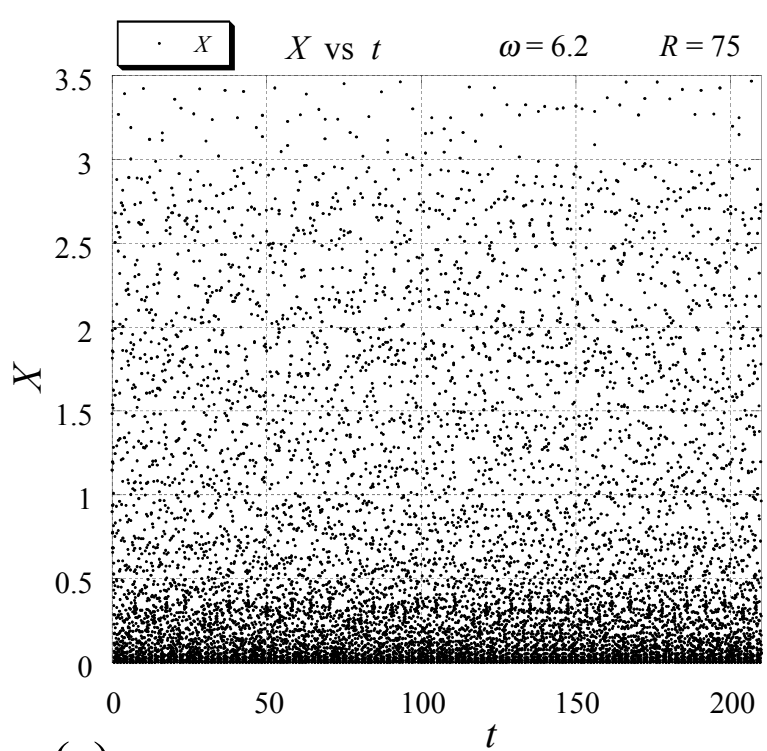

(a)

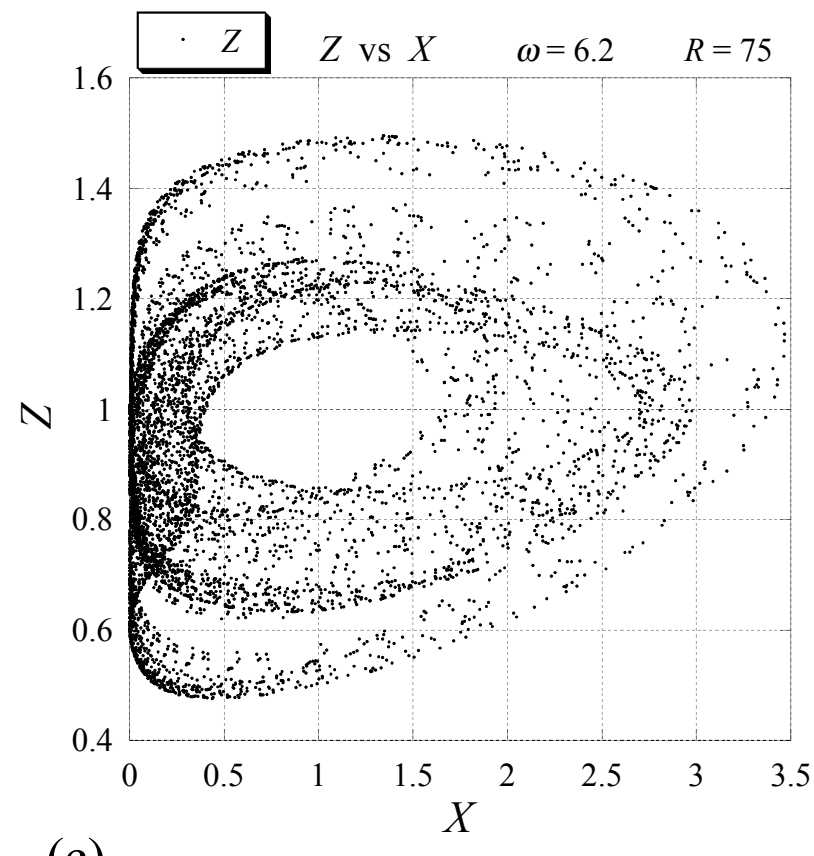

(c)
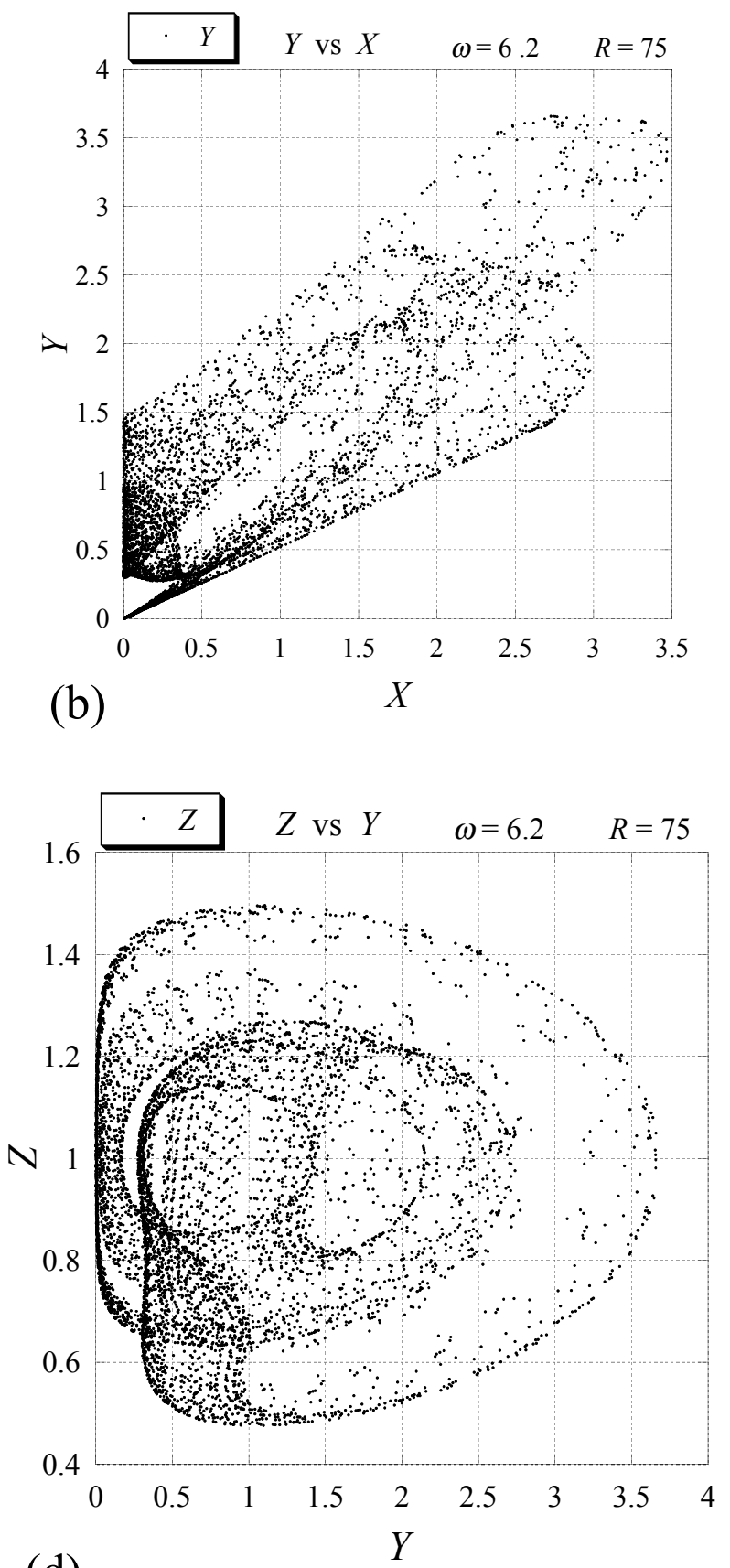

(d)

Figure 6: Graphical description of the solution for $\omega=6.2$ and $R=75$; (a) the amplitude solution in the time domain $X(t)$; (b), (c), and (d) the solution on the phase diagram: projection of the trajectory on the $X-Y, X-Z$, and $Y-Z$ planes, respectively. 
Figure 7

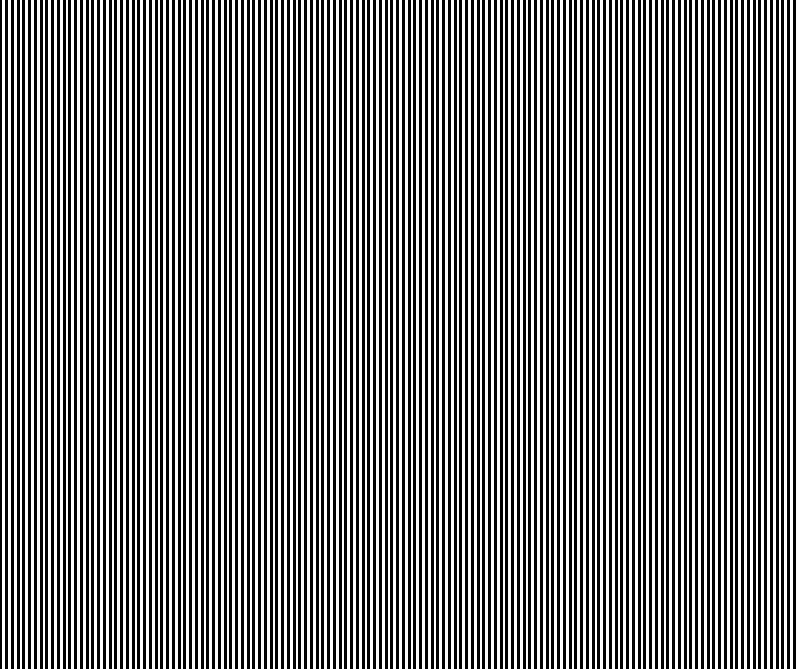

(a)

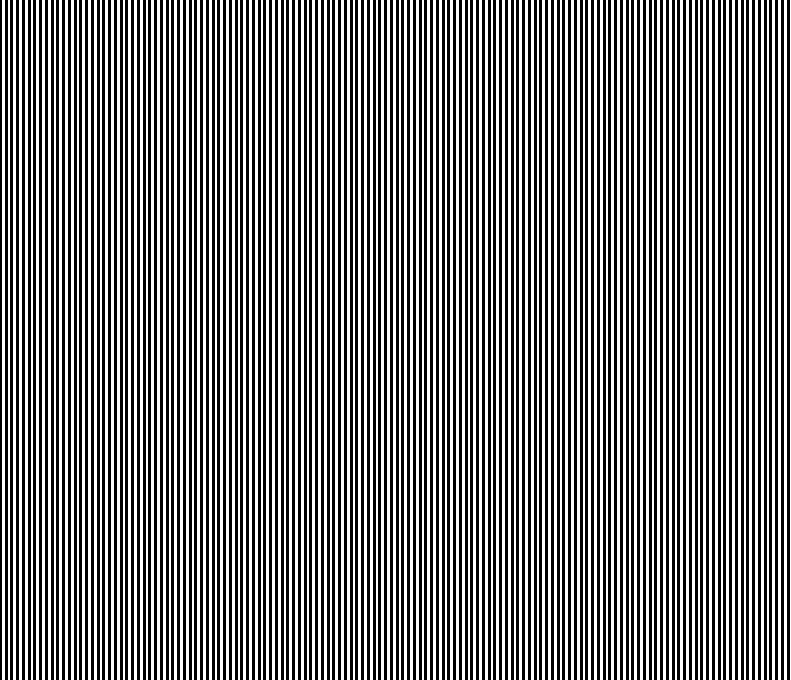

(c)

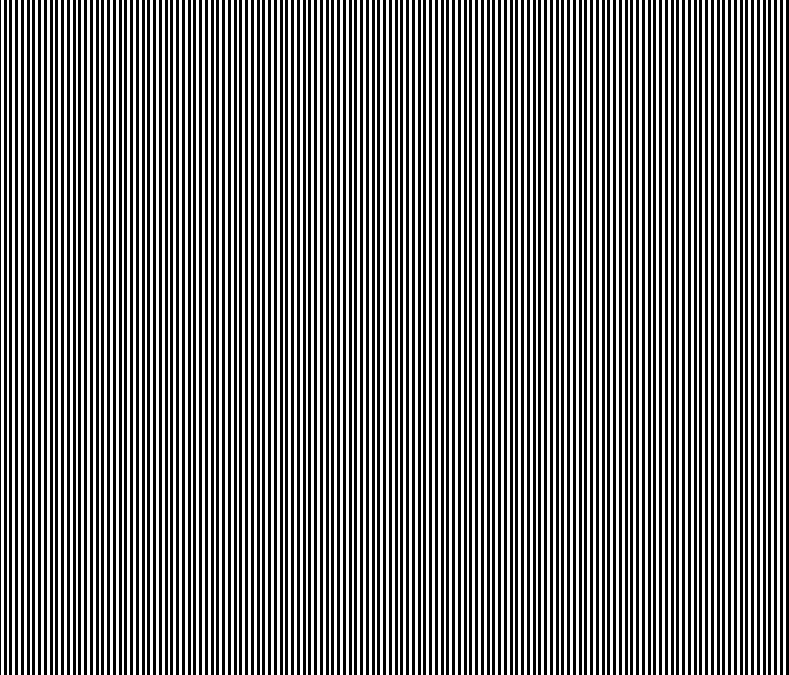

(b)

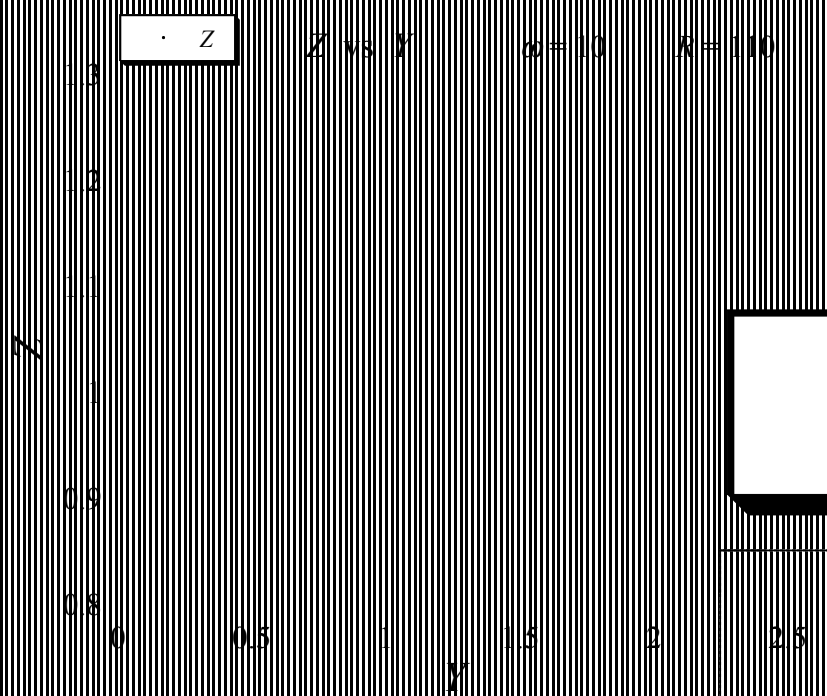

(d)

Figure 7: Graphical description of the solution for $\omega=10$ and $R=110$; (a) the amplitude solution in the time domain $X(t)$; (b), (c), and (d) the solution on the phase diagram: projection of the trajectory on the $X-Y, X-Z$, and $Y-Z$ planes, respectively. 
Figure 8

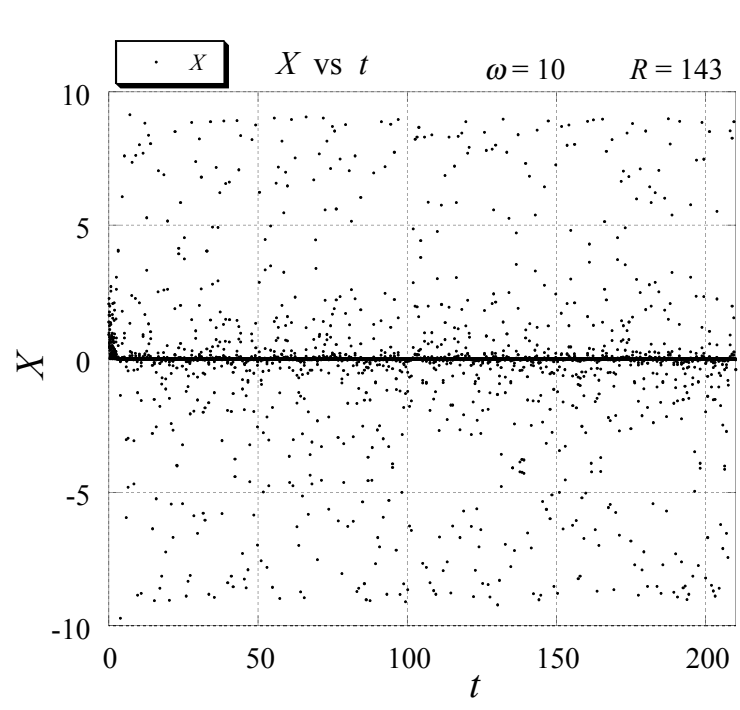

(a)

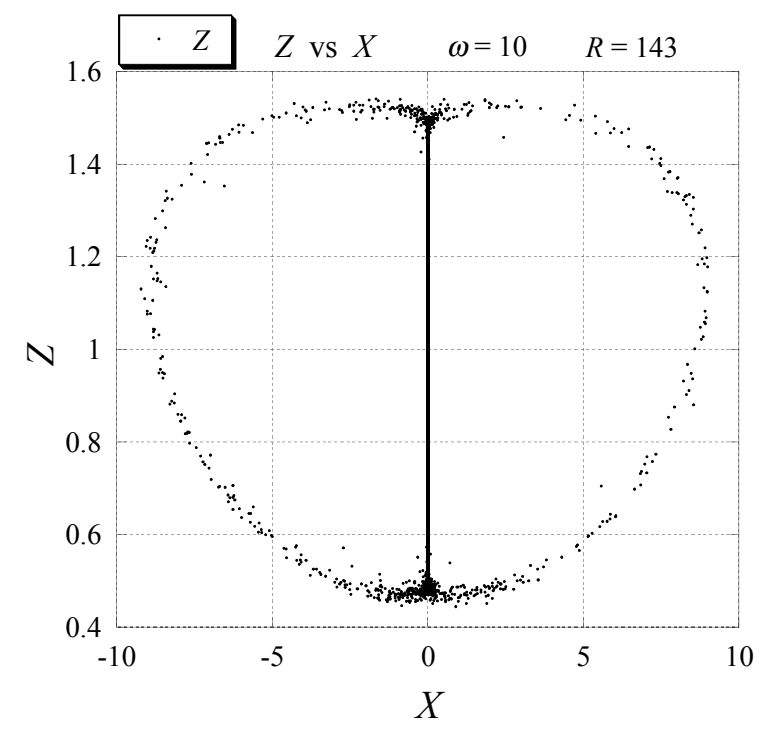

(c)

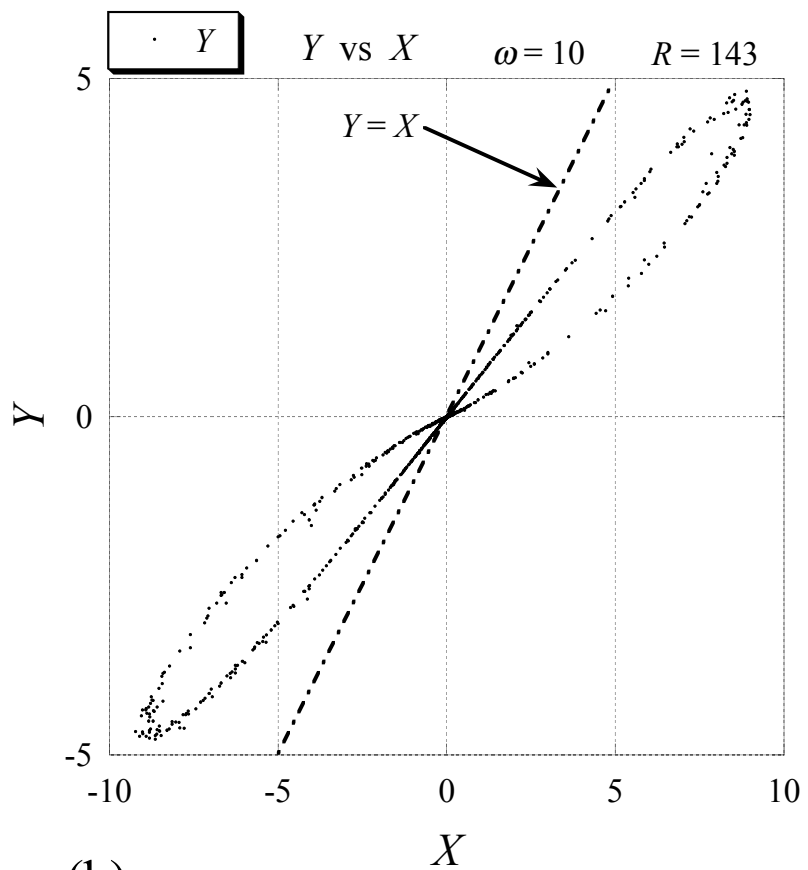

(b)

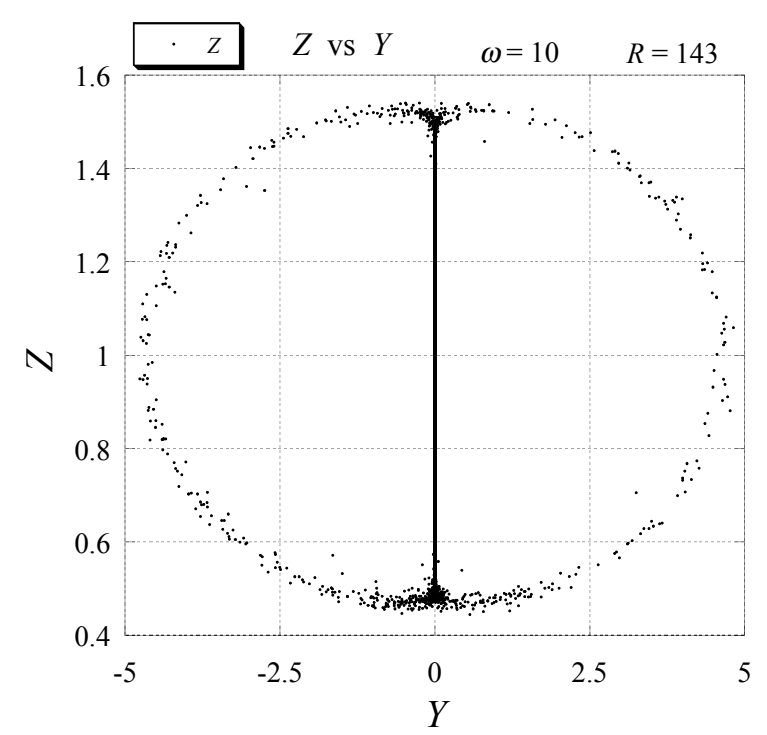

(d)

Figure 8: Graphical description of the solution for $\omega=10$ and $R=143$; (a) the amplitude solution in the time domain $X(t)$; (b), (c), and (d) the solution on the phase diagram: projection of the trajectory on the $X-Y, X-Z$, and $Y-Z$ planes, respectively. 
Figure 9

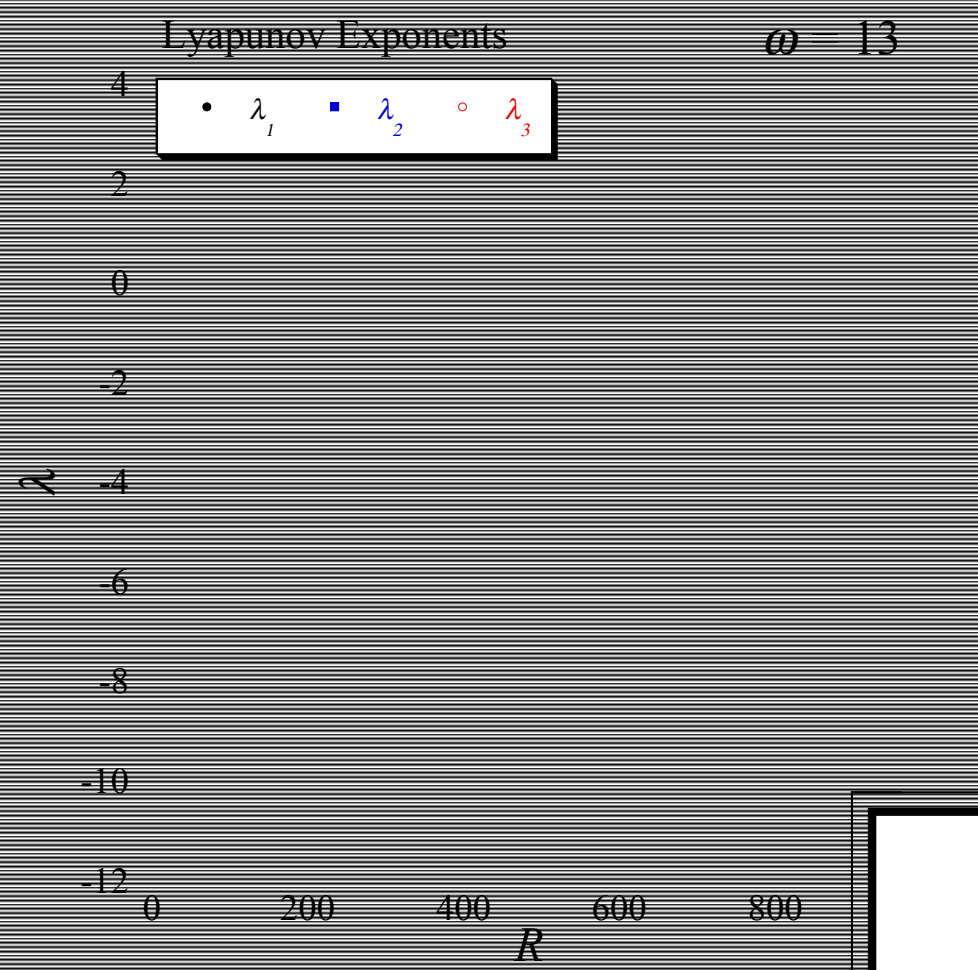

Figure 9: Lyapunov exponents plotted as a function of $R$ for $\omega=13$.

Figure 10

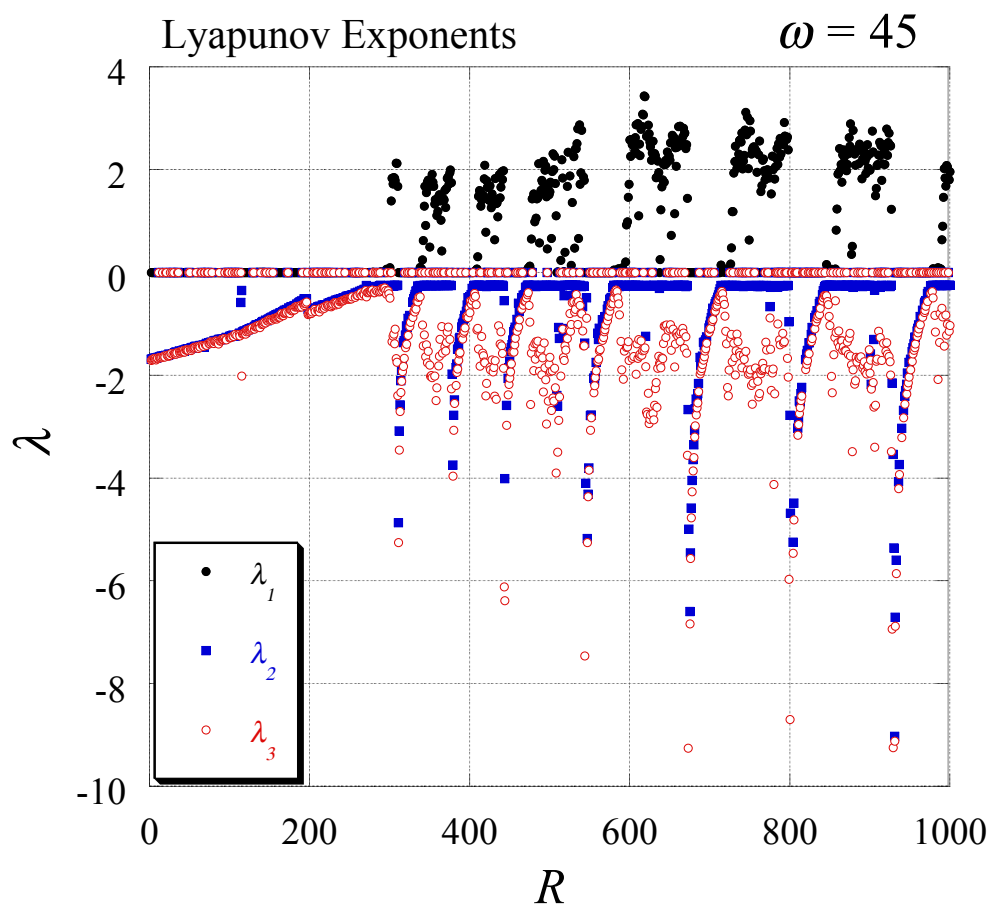

Figure 10: Lyapunov exponents plotted as a function of $R$ for $\omega=45$. 


\section{REFERENCES}

Bhadauria, B.S.: Double diffusive convection in a porous medium with modulated temperature on the boundaries. Transport in Porous Media 70 (2), 191-211 (2007).

Bhadauria, B.S., Kiran, P.: Heat Transport in an Anisotropic Porous Medium Saturated with Variable Viscosity Liquid Under Temperature Modulation. Transport in Porous Media 100, 279-295 (2013).

Bardan, G., Mojtabi, A.: On the Horton-Rogers-Lapwood convective instability with vertical vibration: onset of convection. Physics of Fluids 12, 2723-2731 (2000).

Bejan, A.: Convection Heat Transfer. (2nd edition) Wiley, New York, (1995).

Charrier-Mojtabi, M.C., Elhajjar, B., Mojtabi, A.: Analytical and numerical stability analysis of Soretdriven convection in a horizontal porous layer. Physics of Fluids 19 (124104/1-14), (2007).

Elhajjar, B., Mojtabi, A., Charrier-Mojtabi, M.C.: Influence of vertical vibrations on the separation of a binary mixture in a horizontal porous layer heated from below. International Journal of Heat and Mass Transfer 52, 165-172 (2009).

Govender, S.: Stability of convection in a gravity modulated porous layer heated from below. Transport in Porous Media 57, 113-123 (2004).

Govender, S.: Linear stability and convection in a gravity modulated porous layer heated from below: transition from synchronous to subharmonic solutions. Transport in Porous Media 59, 227-238 (2005).

Govender, S.: Destabilising a fluid saturated gravity modulated porous layer heated from above. Transport in Porous Media 59, 215-225 (2005).

Govender, S.: Stability analysis of a porous layer heated from below and subjected to low frequency vibration: frozen time analysis. Transport in Porous Media 59, 239-247 (2005).

Govender, S.: Weak non-linear analysis of convection in a gravity modulated porous layer. Transport in Porous Media 60, 33-42 (2005).

Govender, S.: Stability of gravity driven convection in cylindrical porous layer subjected to vibration. Transport in Porous Media 63 (2006) 489-502.

Govender, S.: Vadasz number influence on vibration in a rotating porous layer placed far away from the axis of rotation. ASME J Heat Transfer 132, (112601/1-5) (2010).

Govender, S.: Stability of moderate Vadasz number solutal Convection in a cylindrical mushy layer subjected to vertical vibration. Transport in Porous Media 88, 225-234 (2011).

Govender, S.: Natural convection in gravity-modulated porous layers, in Emerging topics in heat and mass transfer in porous media - theory and applications of transport in porous media. P. Vadasz (Ed.) 22, 133-148 (2008).

Hull, T.E., Enright, W.H., Jackson, K.R.: User's Guide for DVERK: a Subroutine for Solving Non-Stiff ODE's. Rept.100, Department of Computer Science, University of Toronto, Canada, 1976.

Jounet, A., Bardan, G.: Onset of thermohaline convection in a rectangular porous cavity in the presence of vertical vibration. Physics of Fluids 13 (11), 3234-3246 (2001).

Lombardo, S., Mulone, G.: Necessary and sufficient conditions of global nonlinear stability for rotating double-diffusive convection in a porous medium. Continuum Mech. Thermodyn. 14, 527-540 (2002).

Lorenz, E.N.: Deterministic non-periodic flows. Journal of the Atmospheric Sciences 20 130-141, (1963).

Magyari, E.: The "Butterfly Effect" in a porous slab. Transport in Porous Media 84 (3), 711-715 (2010).

Magyari, E.: The Vadasz-Olek model regarded as a system of coupled oscillators. Transport in Porous Media 85 (2), 415-435 (2010).

Mahmud, M.N., Hashim, I.: Small and moderate Prandtl number chaotic convection in porous media in the presence of feedback control. Transport in Porous Media 84 (2), 432-440 (2010). 
Mahmud, M.N., Hashim, I.: Small and moderate Vadasz number chaotic convection in porous media in the presence of non-Boussinesq effects and feedback control. Physics Letters A 375, 2382-2393, (2011).

Mulone, G. and Straughan, B.: An operative method to obtain necessary and sufficient stability conditions for double diffusive convection in porous media. Z. Angew. Math. Mech. ZAMM 86 (7), 507-520 (2006)

Nield, D.A., \& Bejan, A.: Convection in Porous Media. $3^{\text {rd }}$ Edition, Springer Verlag, New York (2006).

Roslan, R., Mahmud, M.N., Hashim, I.: Effects of feedback control on chaotic convection in fluidsaturated porous media. International Journal of Heat and Mass Transfer 54 (1-3), 404-412 (2011).

Sheu, L.J.: An autonomous system for chaotic convection in a porous medium using a thermal nonequilibrium model. Chaos, Solitons \& Fractals 30 (3), 672-689 (2006).

Sparrow, C.: The Lorenz Equations: Bifurcations, Chaos, and Strange Attractors. Springer-Verlag, New York, (1982).

Straughan, B.: A sharp nonlinear stability threshold in rotating porous convection. Proc. Royal Society of London A 457 (No. 2005), 87-93 (2001)

Straughan, B.: Resonant porous penetrative convection. Proc. Royal Society of London A 460, 29132927 (2004).

Vadasz, J.J., Meyer, J.P., Govender, S.: Vibration effects on weak turbulent natural convection in a porous layer heated from below. Int. Comm. Heat Mass Transfer 45, 100-110 (2013).

Vadasz, P.: Coriolis Effect on Gravity Driven Convection in a Rotating Porous Layer Heated from Below. Journal of Fluid Mechanics 376, 351-375 (1998).

Vadasz, P.: Local and global transitions to chaos and hysteresis in a porous layer heated from below. Transport in Porous Media 37 (2), 213-245 (1999).

Vadasz, P.: Subcritical transitions to chaos and hysteresis in a fluid layer heated from below. International Journal of Heat and Mass Transfer 43 (5), 705-724 (1999).

Vadasz, P.: Heat transfer regimes and hysteresis in porous media convection. ASME J Heat Transfer 123, 145-156 (2001).

Vadasz, P.: Controlling chaos in porous media convection by using feedback control. Transport in Porous Media 85 (1), 287-298 (2010).

Vadasz, P., Olek, S.: Weak turbulence and chaos for low Prandtl number gravity driven convection in porous media. Transport in Porous Media 37 (1), 69-91 (1999).

Vadasz, P., Olek, S.: Route to chaos for moderate Prandtl number convection in a porous layer heated from below. Transport in Porous Media 41 (2), 211-239 (2000).

Vafai, K., Hadim, H.: Overview of current computational studies of heat transfer in porous media and their applications - natural and mixed convection. Ch. 10, in: W.J. Minkowycz, E.M. Sparrow (Eds.), Advances in Numerical Heat Transfer, 2, Taylor \& Francis, New York, 2000. 